Original Research Paper

\title{
Fungal Concentrations in Dust Samples of Animal Rearing Houses of Bangalore, India
}

\author{
Pavan Rangahanumaiah \\ Department of Microbiology and Biotechnology, Bangalore University, Bangalore-56, India
}

Article history

Received: 10-01-2015

Revised: 08-05-2015

Accepted: 28-05-2015

\begin{abstract}
Fungal concentrations in dust samples analyzed by molecular methods were carried out of animal rearing house at Hessaraghatta village, Bangalore. Handy air sampler EnviroTech-APM 821 was used to collect the dust samples in triplicate and sampling time was limited to eight hours. The sampled filter papers were used for the assay of DNA extracted from all the four animal rearing locations were used for PCR amplification using Universal ITS fungal identification primers PCR products were analyzed on $2 \%$ agarose gel out of four different locations were rabbit house, cow shed, poultry farm and swine house. The sequencing was then blasted against known sequences in the NCBI database to provide species identification and phylogenetic tree was also constructed with 20 closely related blast sequences. The rabbit house and cow shed dust samples did not show any amplifications even with varying conditions while poultry farm (Cladosporium oxysporium DFFSCS018) and swine house (Peniophora species1306-hpF-04) isolate samples gave amplicons of 550bp and 600bp respectively with ITS region showing $99 \%$ similarity. These studies useful for clinicians to identify the cause of the dust allergic diseases of the animal rearing house workers and suggest amendment strategies.
\end{abstract}

Keywords: Animal, Dust, Fungi, Sequence

\section{Introduction}

Recent rapid advances in molecular biology provide the potential for enhanced characterization and rapid quantification of microorganisms in the environment. Application of advanced molecular techniques will greatly enhance the speed, quality and quantity of data generation, leading to enhanced process understanding and major improvements to predictive models which are now being opened up and recommended as regulatory tools by World Health Organisation for management in certain model systems. Prediction of exposure is the key to protection of the public, with real-time molecular measurements providing a valuable back-up and model calibration tool. Molecular methods, including quantitative PCR and full genome sequencing are capable of rapid speciation and enumeration of organisms.

Polymerase Chain Reaction (PCR) technique has been used to detect and quantify microorganisms from various environments (Gilbert et al., 2010). It is used to copy and amplify many million-fold specific regions (typically $<1000$ bases) of the genome, providing enough for analyses (Georgakopoulos et al., 2009). This method is very rapid compared to that with culture techniques and is sensitive enough for the detection of specific microorganisms which are slow growing and difficult to culture. Mainly outdoor microorganisms have been investigated using this method (Dungan et al., 2010). The PCR and DNA hybridization techniques are promising approaches which are complementary to the conventional detection methods. PCR amplification only requires intact cellular DNA of microorganisms present in the sample, regardless of their culturability and viability. PCR and probe hybridization techniques have been demonstrated to be sensitive and accurate for detection of fungi in clinical specimens in indoor environments and in other environmental samples (Zhihong et al., 2002).

Molecular methods based on DNA analysis reveal fungal diversity in ecosystems and offer the potential benefits of highly sensitive and rapid detection (Saad et al., 2004). In fungi, the Internal Transcribed Spacer (ITS) region between the conserved flanking regions of the small and large subunit of ribosomal RNA is the most frequently sequenced genetic marker of fungi and has been used to address research questions relating to systematics, phylogeny and identification of strains at and 
even below the species level and commonly used primers for amplifying the ITS region (Martin and Rygiewicz, 2005). The present studies of animal houses were taken up the identify the airborne fungal spores in dust collected from the animal rearing house of Bangalore.

\section{Materials and Methods}

The sampling site selected for fungal concentrations in dust samples of animal rearing houses were analyzed by PCR methods were Location 1 - Rabbit house, Location 2 Cow shed, Location 3 - Poultry farm and Location 4 Swine house at Hessaraghatta village, Bangalore in the State of Karnataka, India. For the assay of DNA extraction, a Handy air sampler Envirotech APM 821 was used to collect the dust samples in triplicate. Preweighed glassfibre filter papers (Whatmann, $25 \mathrm{~mm}$ diameter) were used. Sampling was done for eight hours. The filter disc was carefully removed with the help of flat tipped sterile forceps and the disc was then placed in zip lock freezer bags.

The samples were used for the assay of DNA extraction and the quality of the fungal DNA that was extracted was analyzed on $0.8 \%$ agarose gel and visualized under UV Gel documentation system. The forward (ITS-1) and reverse (ITS-4) primers used in the PCR reactions performed in this study were developed by White et al., (1990) and are designed to amplify the ITS region of the rRNA operon.

Polymerase chain reaction products were purified following the protocols of the QIA quick PCR Purification Kit (Qiagen, Valencia, CA, USA). The purified DNA was eluted from spin columns with $30 \mu$ of nuclease free $\mathrm{H}_{2} \mathrm{O}$ and DNA concentrations were determined with a spectrophotometer. The sequences were then blasted against known sequences in the National Center for Biotechnology Information database to provide species identification and phylogenetic tree was also constructed with 20 closely related blast sequences.

\section{Results}

\section{PCR Based Detection and Quantification of Fungal Spores in Dust Extracts}

DNA was isolated from the dust samples of four different animal rearing site and all the DNA samples were analyzed on $0.8 \%$ agarose gel and visualized under UV gel Documentation unit (Fig. 1). DNA extracted from all the four locations were used for PCR amplification using Universal ITS fungal identification primers and PCR products were analyzed on $2 \%$ agarose gel out of four different locations (Fig. 2). Location 1 and 2 i.e., Dust samples from rabbit house and cow shed did not show any amplifications even with varying conditions while Location 3 and Location 4 i.e., Poultry and swine house samples gave amplicons of 550bp and
$600 \mathrm{bp}$ respectively. The amplicons were purified and was sequenced using ITS primers.

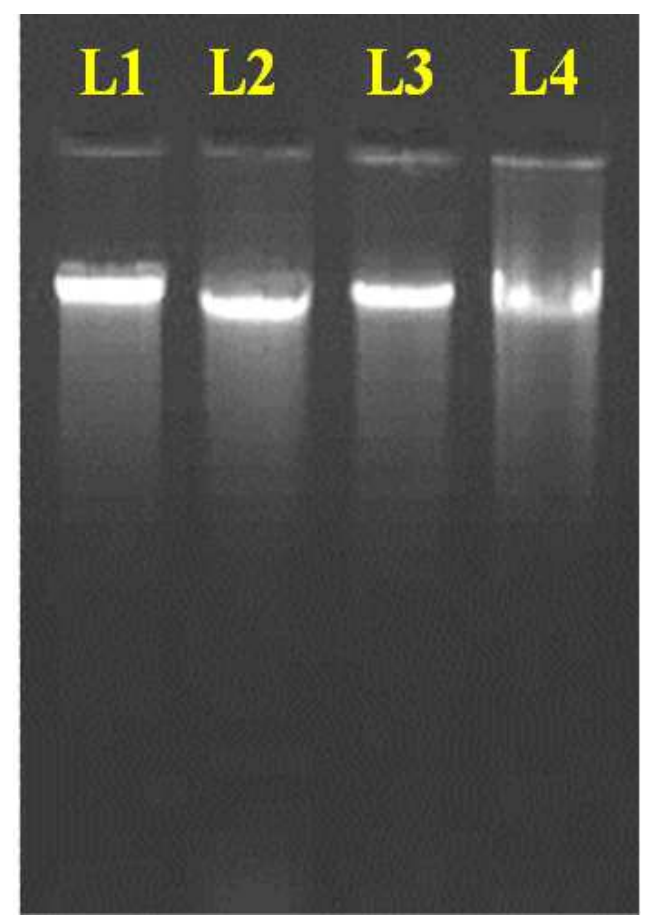

Fig. 1. Qualitative analysis of fungal Genomic DNA on $0.8 \%$ agarose gel from dust samples

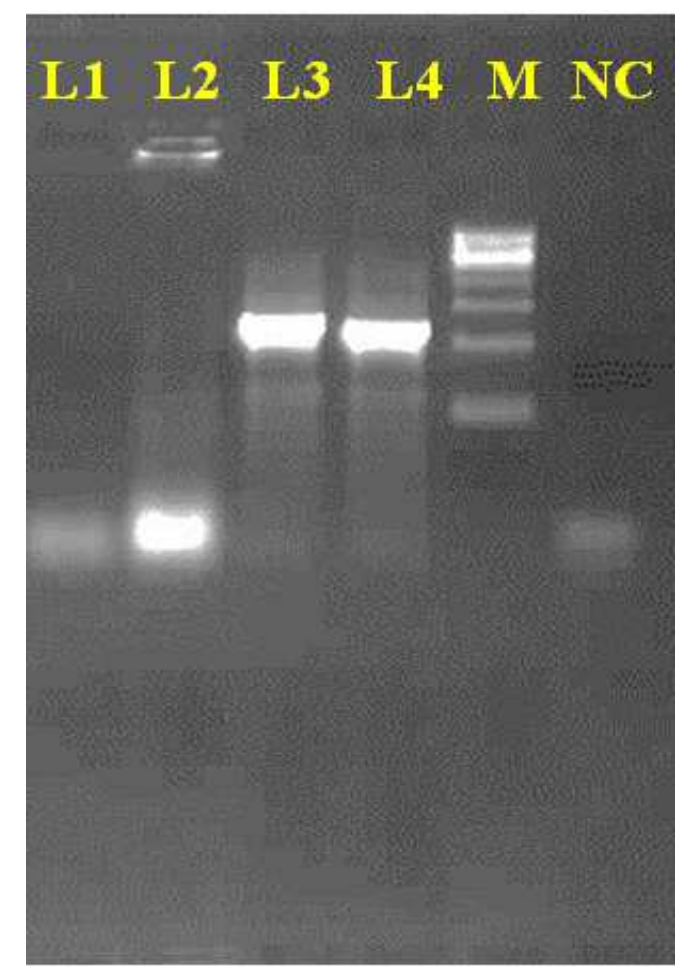

Fig. 2. PCR amplicons using fungal ITS primers on $2 \%$ agarose gel 


\begin{tabular}{|c|c|}
\hline \multicolumn{2}{|l|}{ 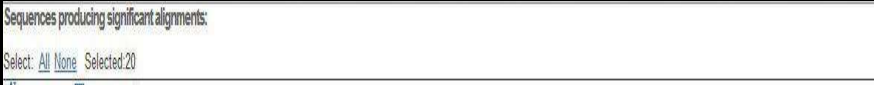 } \\
\hline 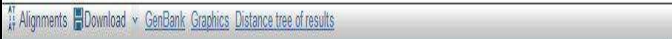 & \\
\hline Descinition & 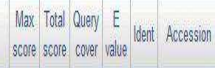 \\
\hline 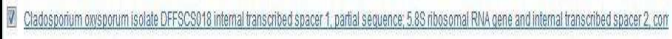 & 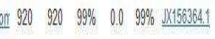 \\
\hline 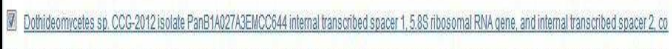 & $0292092099 \% 00.099 \%$ \\
\hline 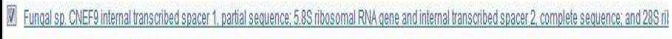 & 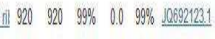 \\
\hline 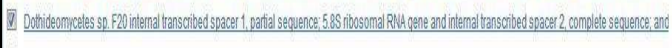 & 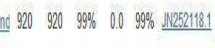 \\
\hline 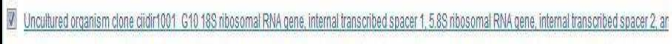 & 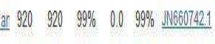 \\
\hline 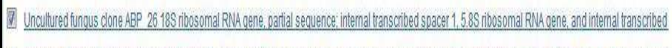 & 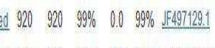 \\
\hline 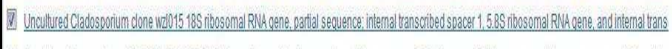 & $1992092099960.099 \%$ \\
\hline 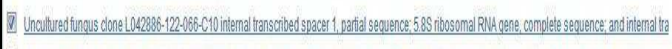 & tag $920922999 \% 0.099 \% \underline{628516711}$ \\
\hline 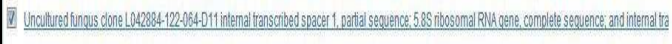 & tha $92092099 \%$ on $99 \% 6$ \\
\hline 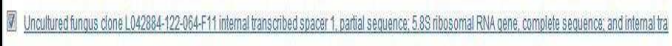 & 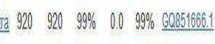 \\
\hline 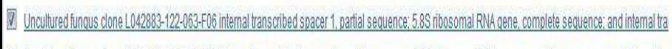 & 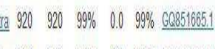 \\
\hline 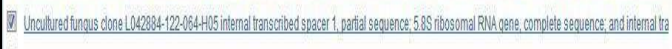 & 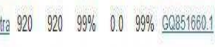 \\
\hline 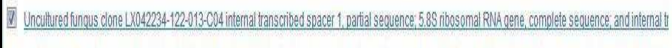 & 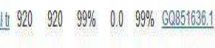 \\
\hline 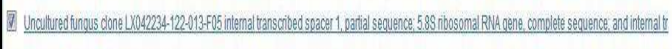 & $11920920999 \% 009996$ \\
\hline 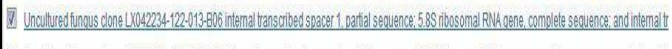 & $1192092099 \% 0.099 \% 6208516221$ \\
\hline 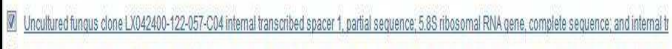 & $102092099 \%$ 0.0 $99 \%$ \\
\hline 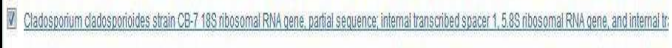 & 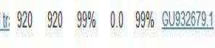 \\
\hline 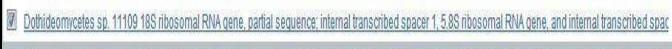 & $2920920999 \%$ 0.0 $99 \% \underline{601550021}$ \\
\hline 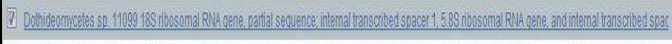 & 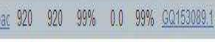 \\
\hline 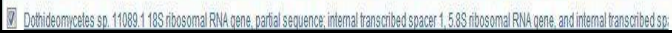 & $592092099 \% 00998 \%$ \\
\hline
\end{tabular}

Fig. 3. NCBI BLAST results showing distance tree using Neighbour joining in Newick method

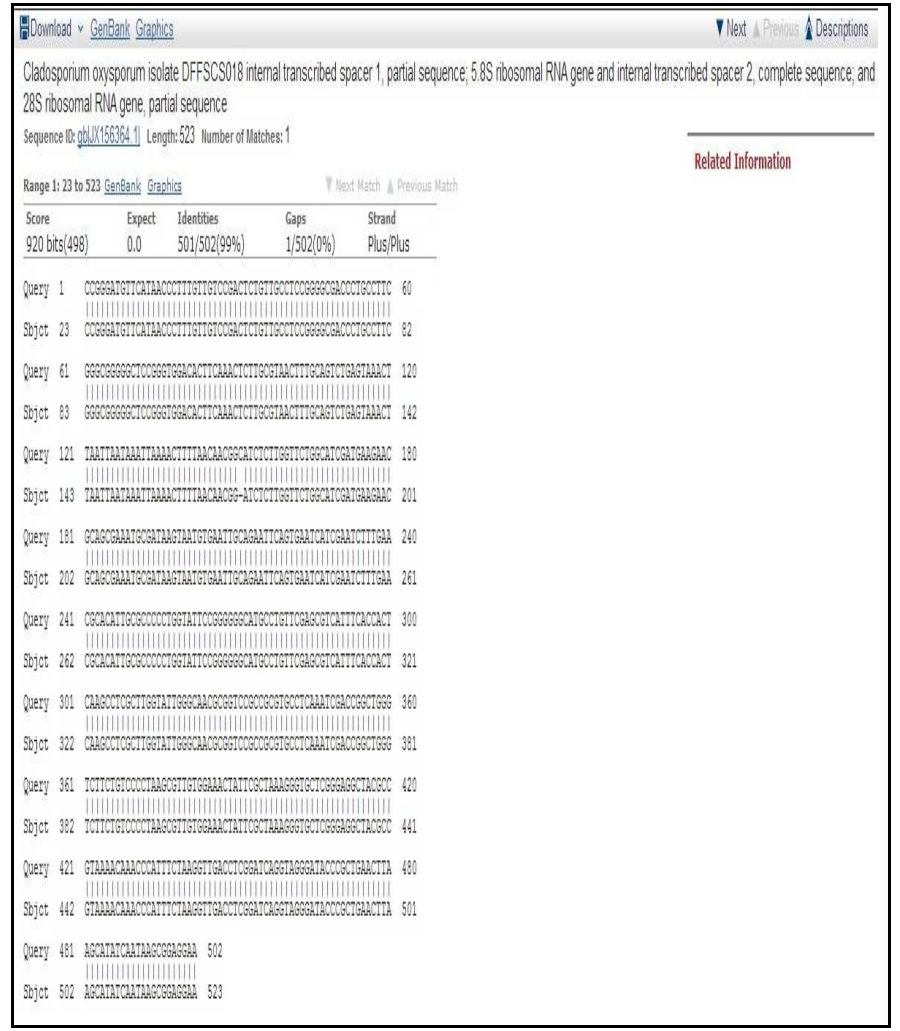

Fig. 4. Cladosporium oxysporium isolate DFFSCS018 ITS region showing 99\% similarity with the poultry dust samples isolate 


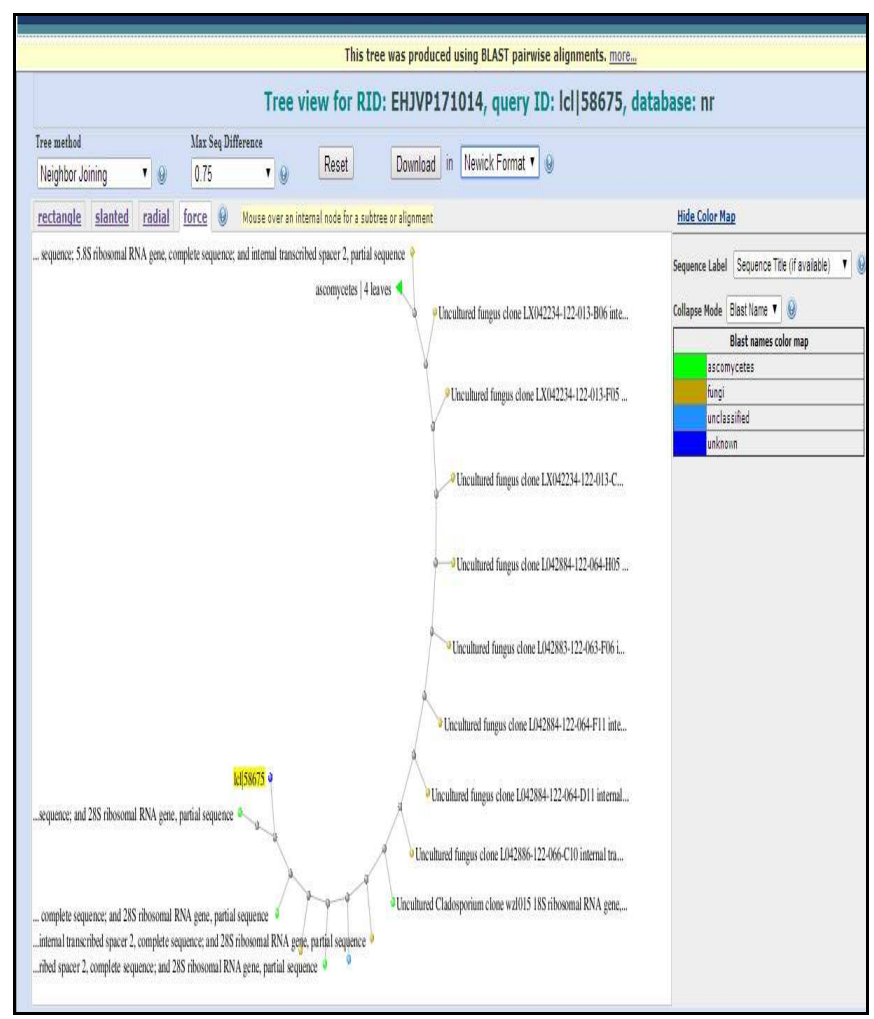

Fig. 5. Distance tree using Neighbour joining in Newick method



Fig. 6. NCBI BLAST results showing distance tree using Neighbour joining in Newick method 


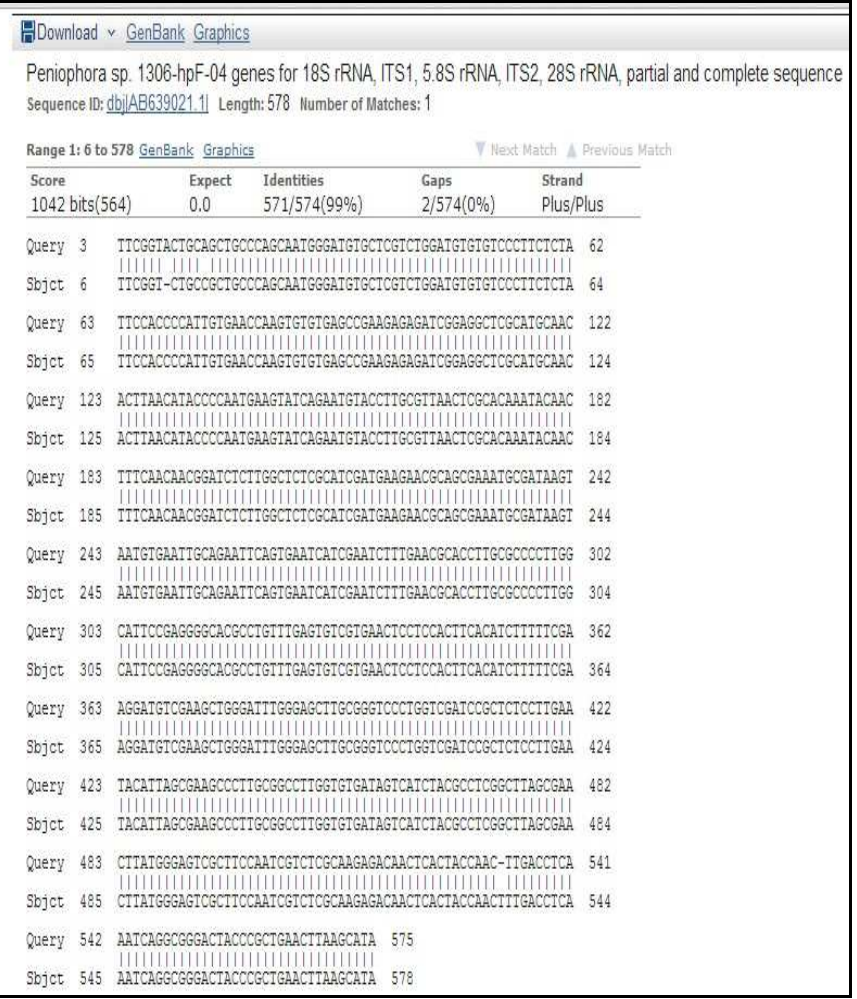

Fig. 7. Peniophora species1306-hpF-04 ITS region showing 99\% similarity with the Swine house dust samples

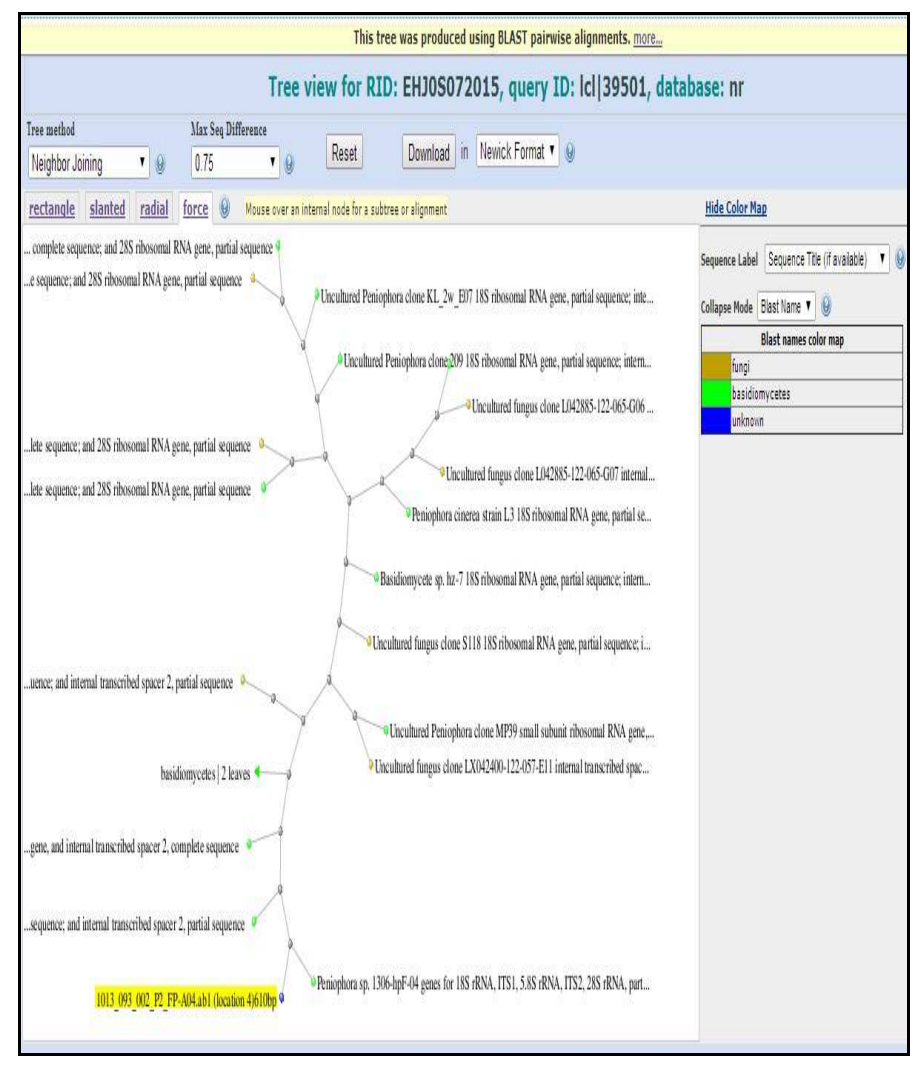

Fig. 8. Distance tree using Neighbour joining in Newick method 
DNA Sequencing of Poultry Farm Dust Samples $>$ 1013_093_001_P1_FP-H03.ab1 (location 3)504bp

The sequence was blasted with the NCBI Nucleotide blast database (Fig. 3). To identify the fungal species 20 closely related species (Fig. 4) and that were produced in BLAST were used to construct the distance tree using Neighbor joining in Newick method (Fig. 5).

DNA Sequencing of Swine House Dust Samples >1013_093_002_P2_FP-A04.ab1 (Location 4)610bp

The sequence was blasted with the NCBI Nucleotide blast database (Fig. 6). To identify the fungal species 20 closely related species (Fig. 7) and that were produced in BLAST was used to construct the distance tree using Neighbor joining in Newick method (Fig. 8).

\section{Discussion}

The present study carried out relative occurrence of airborne fungal concentrations in dust samples of animal rearing houses viz., rabbit house, cow shed, poultry farm and swine house in Hessaraghatta village, Bangalore were analyzed by PCR methods. There are few reports on the airborne fungi and fungal aeroallergens (Kullman et al., 1998) of organic dusts including airborne fungi (Duchaine et al., 1999). This study gave rough approximation and qualitative information about the types and concentrations of the various airborne fungi in organic dust samples also this study useful to determine the effectiveness of using PCR to amplify the ITS region of fungal DNA for the purposes of sequencing and eventual species identification.

PCR studies for nonviable fungi revealed that traditional methods of identifying fungi can be extremely difficult and may result in a species list that misrepresents the fungal community. However, molecular techniques that allow for the comparison of DNA sequence information between known and unknown fungal species is much simpler and more reliable than traditional methods and has revolutionized the field of fungal ecology. The technique proved successful as its application led to the identification of fungi in the dust samples to the species level. In this study successful amplification was possible only for two of the locations that are poultry farm and swine house while the other two locations did not give any amplicons even with varying conditions. Single distinct band was fractionated on a $2 \%$ agarose gel after PCR amplification of the dust samples. However, agarose gel did not provide sufficient resolution to successfully separate bands produced by fungi with very similarly sized ITS regions, such as for Cladosporium oxysporium, Dothideomycetes species, Cladosporium cladosporioides from poultry farm and Peniophora lycii, Amantia from swine house samples. In these situations, all products migrated to the same region of the gel (550 bp in poultry farm) and (610 bp in swine house) when run in the same well. Therefore, one can say that single bands produced from a mixed sample of unknown fungi contain multiple species.

This method of identification of fungal species will mainly help in identification of unculturable fungal species that are sometimes harmful and sometimes also pose media constraints for culturing such fungal species. Identification of fungi from environmental samples as bands could simply be excised from the gel and sequenced. More intensive screening of fungal species using this technique could eventually lead to the compilation of a fungal species barcode in which sequencing the bands would no longer be necessary. A distance tree was also constructed using the same sequences respectively in NCBI database for which the first 20 matched sequences was taken for consideration along with the amplified sequences, which produced a distance tree that showed a very close relationship with Cladosporium oxysporum followed by Dothiodeomycetes. These result indicates dominating population of the above two species in the poultry farm while a very close relationship was observed with Peniophoralycii followed by Amatia species, indicating dominating population of the above two species in the swine house.

Comparing the findings of different studies on airborne fungi is difficult since the spore concentrations depends on different climatic conditions, vegetations and fungal growth substrates. In addition to the variety of sampling methods used by the various investigations, a similar opinion has been expressed by Aydogdu and Asan (2008).

There are loads of dusts and fungal spores present in animal rearing house air. There seem to be a health risk for animal and man indoor caused by these substances. These substances are emitted in considerable amounts from buildings and manure stores into the environment. There is still a considerable lack of knowledge on the distribution and health effects of airborne fungi particulate emissions from livestock sources in the environment. For licensing new animal farms as well as residential areas in the farming environment more precise information on the travel distance of harmful particles and compounds are required. For the realization of these aims the cooperation of farmers, agricultural engineers, veterinarians and governmental agencies is necessary. It was found through this conclusion that the fungal concentration in breeding house is higher than outdoor and indoor environment. The concentration could be reduced by way of choosing opened or semi-blocked structure animal house. Adjusting the temperature and humidity could control fungal concentration. The airborne fungal 
spores can be easily inhaled into the deep respiration tract than bacterium. The fungal concentrations of environment were changed with function of the places and the dominant fungus has close relation.

\section{Conclusion}

The results showed that the airborne fungi size was very closely correlated with the endangerment of airborne fungal dust particles as well. Aerosol fungal concentrations were influenced by many factors such as animal habit, weather, sanitation condition and illumination. Caretakers at the sampling sites have prolonged exposure to such environment which result in occult infection or develop to chronic nosomycosis and lead to predisposition to other diseases. At present, there are no safe levels of airborne fungi concentration in indoor environments, but high concentration would result in threats to the health of human beings and animals. These studies useful for clinicians to identify the cause of the dust allergic diseases, prescribe necessary treatment for the workers and suggest amendment strategies in the animal rearing house.

\section{Acknowledgement}

Author wish to thank Department of Microbiology and Biotechnology, Bangalore University, Bangalore for providing laboratory facilities.

\section{Funding Information}

Author acknowledge the financial support provided under University Grant Commission-Basic Science Research (UGC-BSR) Programme, New-Delhi, India.

\section{Ethics}

This article is original and contains unpublished material. The corresponding author confirms that have read and approved the manuscript and no ethical issues involved.

\section{References}

Aydogdu, H. and A. Asan, 2008. Airborne fungi in child day care centers in Edirne City, Turkey. Environ. Monit. Assess., 147: 423-444.

DOI: $10.1007 / \mathrm{s} 10661-007-0130-4$

Duchaine, C., Meriaux, A., Brochu, G. and Cormier, Y. 1999. Airborne microflora in Quebec dairy farms: lack of effect of bacterial hay preservatives. Am. Industrial Hygiene Association J., 60: 89-95.

DOI: $10.1080 / 00028899908984426$
Dungan, R.S., A.B. Leytem, S.A. Verwey and D.L. Bjorneberg, 2010. Assessment of bioaerosols at a concentrated dairy operation. Aerobiologia, 26: 171-184. DOI: 10.1007/s10453-010-9154-2

Georgakopoulos, D.G., V. Després, J. FröhlichNowoisky, R. Psenner and P.A. Ariya et al., 2009. Microbiology and atmospheric processes: biological, physical and chemical characterization of aerosol particles. Biogeosciences, 6: 721-737. DOI: $10.5194 /$ bg-6-721-2009

Gilbert, Y., M. Veillette and C. Duchaine, 2010. Airborne bacteria and antibiotic resistance genes in hospital rooms. Aerobiologia, 26: 185-194. DOI: $10.1007 / \mathrm{s} 10453-010-9155-1$

Kullman, G.J., P.S. Thorne, P.F. Waldron, J.J. Marx and B. Ault et al., 1998. Organic dust exposures from work in dairy barns. Am. Ind. Hygiene Association J., 59: 403-413.

DOI: $10.1080 / 15428119891010668$

Martin, K.J. and P.T. Rygiewicz, 2005. Fungal-specific PCR primers developed for analysis of the ITS region of environmental DNA extracts. BMC Microbiol, 5: 1-11. DOI: 10.1186/1471-2180-5-28

Saad, D.S., G.C. Kinsey, S. Kim and C.C. Gaylarde, 2004. Extraction of genomic DNA from filamentous fungi in biofilms on water-based paint coatings. Int. Biodeterioration Biodegradation, 54: 99-103. DOI: 10.1016/j.ibiod.2004.05.003

White, T.J., T. Bruns, S. Lee and J. Taylor, 1990. Amplification and Direct Sequencing of Fungal Ribosomal RNA Genes for Phylogenetics. In: PCR Protocols: A Guide to Methods and Applications, Innis, M.A., D.H. Gelfand, J.J. Shinsky and T.J. White (Eds.), Publisher Academic Press, San Diego, pp: 315-322.

Zhihong, W., B. Goran, O.W. Sven and R.W. Xiao, 2002. Application of PCR and probe hybridization techniques in detection of airborne fungal spores in environmental samples. J. Environ. Monit., 4: 673-678.

DOI: $10.1039 / B 203048 A$ 